Pathologe 2022 - 43:51-56

https://doi.org/10.1007/s00292-021-01041-5

Angenommen: 15. November 2021

Online publiziert: 23. Dezember 2021

๑) Der/die Autor(en) 2021

Schwerpunktherausgeber

H. I. Grabsch, Maastricht, Niederlande

R. Langer, Linz, Österreich

M. Vieth, Bayreuth

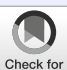

\section{Grading der Tumorregression gastrointestinaler Karzinome nach neoadjuvanter Therapie}

\author{
Drolaiz Liu ${ }^{1,2} \cdot$ Rupert Langer' \\ ${ }^{1}$ Klinisches Institut für Pathologie und Molekularpathologie, Kepler Universitätsklinikum und Johannes \\ Kepler Universität, Linz, Österreich \\ ${ }^{2}$ Department of Pathology, GROW School for Oncology and Developmental Biology, Maastricht University \\ Medical Center+, Maastricht, Niederlande
}

\section{Zusammenfassung}

\section{In diesem Beitrag}

- Histopathologische Befunde nach neoadjuvanter Therapie

- Klassifikation der Tumorregression

- Prognostische Signifikanz der Tumorregression

- Tumorregression bei Lymphknotenmetastasen

- Kritische Punkte der TRG

- Schlussfolgerung und Ausblick

Prä- oder perioperative Chemo- oder Radiochemotherapie und anschließende Resektion ist die Standardtherapie von lokal fortgeschrittenem Ösophagus-, Magen- und Rektumkarzinom. Eine Tumorregressionsgraduierung (TRG, auch Tumorregressionsgrad) kategorisiert das Ausmaß der regressiven Veränderungen nach neoadjuvanter Behandlung. Für gastrointestinale Karzinome existieren mehrere TRG-Systeme, die sich entweder auf das Ausmaß der therapieinduzierten Fibrose im Verhältnis zum Resttumor oder den geschätzten Anteil des Resttumors im Bereich des ehemaligen Tumorareals beziehen. Ein ideales TRG-System zeigt eine signifikante Interobserverübereinstimmung und bietet relevante prognostische Informationen in den meisten Fällen ist eine vollständige oder nahezu vollständige Regression nach neoadjuvanter Therapie mit verbesserter Prognose verbunden. In diesem Review werden die am häufigsten verwendeten TRG-Systeme für gastrointestinale Karzinome vorgestellt und diskutiert. Zudem werden aktuelle Punkte wie die Standardisierung der Angabe von TRGs und die Thematik der Regression bei Lymphknotenmetastasen im Kontext eines TRG-Systems behandelt.

\title{
Schlüsselwörter
}

Gastrointestinale Neoplasien · Histopathologie · Tumorregressionsgrading · Observervariabilität . Referenzstandards

Eine prä- oder perioperative Therapie, typischerweise Chemo- oder Radiochemotherapie, gefolgt von der chirurgischen Resektion ist Standardtherapie für lokal fortgeschrittene gastrointestinale Neoplasien, insbesondere von Ösophagus-, Magen- und Rektumkarzinomen. Dieses Vorgehen zeigte einen Überlebensvorteil für Patienten im Vergleich zu einer alleinigen Operation [4, 18]. Die Auswirkungen einer neoadjuvanten Therapie auf die Primärlokalisation des Tumors können durch makroskopische und insbesondere histopathologische Untersuchung der resezierten Präparate beurteilt werden [2].

In den letzten Jahrzehnten wurden mehrere Systeme für eine Tumorregressionsgraduierung (TRG, auch Tumorregressions- grad) für Ösophagus-, Magen- und Rektumkarzinome vorgeschlagen, die das Ausmaß der regressiven Veränderungen nach neoadjuvanter Therapie kategorisieren [2, $6,17]$. Der TRG liefert sehr wertvolle prognostische Informationen, da in den meisten Fällen eine vollständige oder subtotale Tumorregression nach neoadjuvanter Therapie mit besserer Prognose verbunden ist.

Im Folgenden werden die charakteristischen histopathologischen Befunde, die nach neoadjuvanter Therapie beobachtet werden, beschrieben und die Konzepte von TRG-Systemen, inklusive Beispiele einiger häufig verwendeter TRG-Systeme für Ösophagus-, Magen- und Rektumkarzinome vorgestellt. Zudem werden Themen wie die Standardisierung der Angabe von TRGs und die Regression bei Lymphkno- 

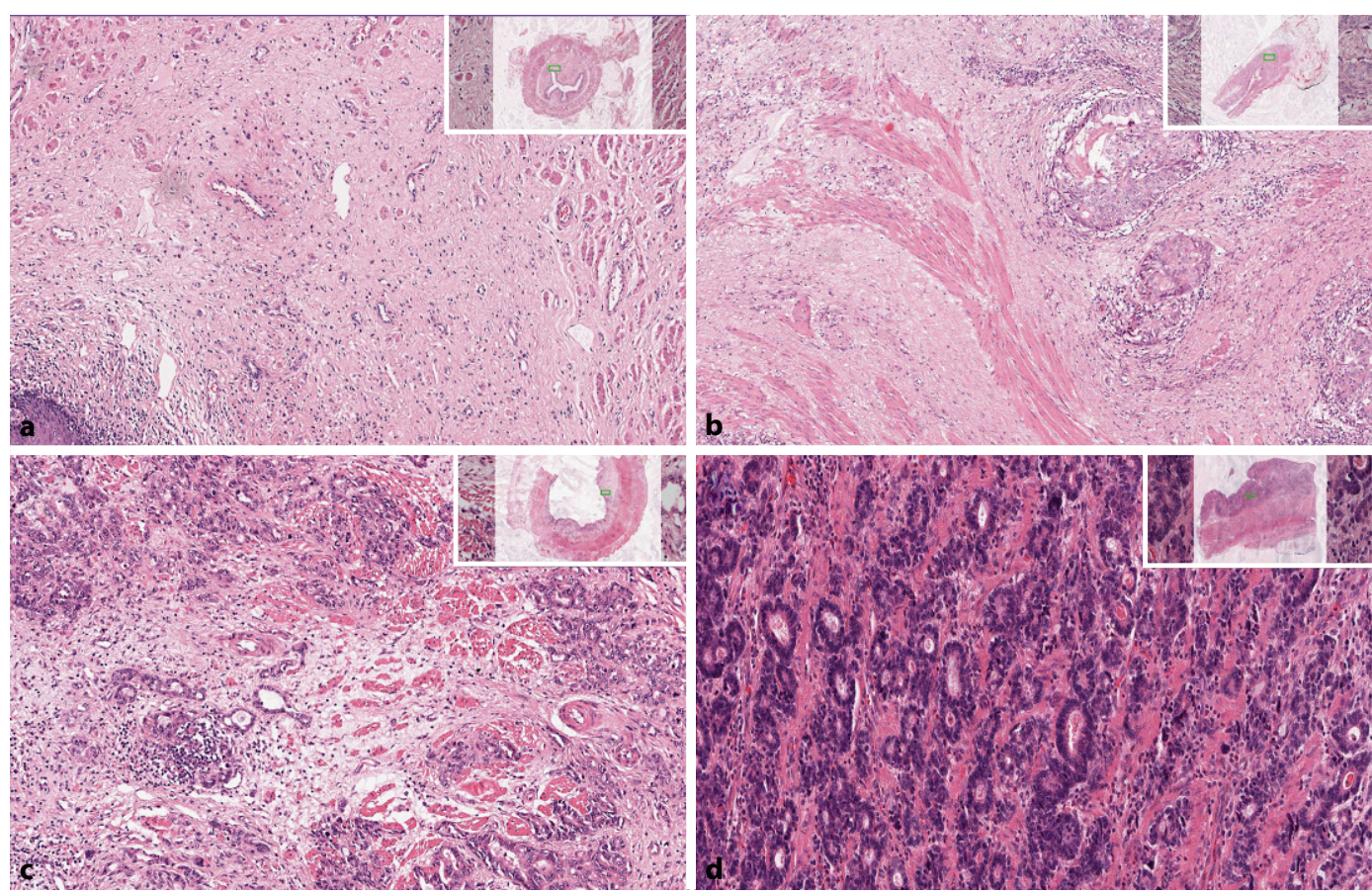

Abb. $1 \varangle$ Verschiedene Kategorien der Tumorregression nach neoadjuvanter Therapie (hier bei gastroösophagealen Karzinomen). a Komplette Regression, b subtotale Regression, c partielle Regression, d wenig oder keine Regression. Die kleinen Einschübe zeigen eine Übersicht über den gesamten Schnitt. Mit der grünen Box ist die Stelle markiert, aus der die vergrößerte Aufnahme stammt tenmetastasen im Kontext eines TRG-Systems behandelt.

\section{Histopathologische Befunde nach neoadjuvanter Therapie}

Der erste Schritt der pathologischen Abklärung ist die makroskopische Beurteilung der Resektionspräparate des Tumors. Hier ist eine erste grobe Abschätzung der Tumorregression möglich, aber noch wichtiger ist es, die Ausdehnung des sogenannten Tumorbetts (der früheren Lage des Tumors) abzuschätzen, um eine korrekte Einbettung für eine genaue histologische Untersuchung zu gewährleisten. Die Tumorregression nach neoadjuvanter Therapie stellt histologisch grundsätzlich eine subakute bis subchronische Entzündung nach zytotoxischer Wirkung dar, die wenige bis mehrere Wochen zuvor eingetreten ist. In den meisten Fällen werden die Tumoren mit einer gewissen Verzögerung nach Abschluss des letzten präoperativen Behandlungszyklus reseziert.

Bei vollständiger Tumorregression werden die Tumorzellen durch die Behandlung und/oder die nachfolgende Entzündungsreaktion zerstört und der Tumor durch fibröses oder fibroinflammatorisches Granulationsgewebe ersetzt. Im Gegensatz dazu kann ein Resttumor entweder reichlich vorhanden sein oder aber nur klei- ne Einzelzellen, tumorbudsähnliche Cluster oder Tumorzellgruppen umfassen. Tumoren können schrumpfen oder fragmentieren [8], sodass die Tumorregression auch einem zentrifugalen Muster folgen kann und der Resttumor nur in der oberflächlichen oder tiefen Peripherie des vorherigen Tumorareals und nicht im Tumorzentrum lokalisiert ist [2]. Resorptive Veränderungen umfassen eine histiozytäre Reaktion mit Schaumzellen oder auch mit Hämosiderin beladenen Makrophagen, Cholesterinspalten und Fremdkörperreaktionen sowie dystrophe Verkalkungen [2]. Bei neoadjuvant behandelten Adenokarzinomen können häufig muzinösen Veränderungen, gelegentlich mit ausgedehnten und teilweise azellulären Muzinseen beobachtet werden [2]. Dieses azelluläre Muzin sollte nicht als vitaler Resttumor betrachtet werden. Keines dieser histologischen Phänomene ist jedoch absolut spezifisch für eine therapieinduzierte Tumorregression und lässt auch per se nicht auf eine vorangegangene Therapie schließen. Anekdotisch ist es in der Routinepraxis auch vorgekommen, dass primär resezierten Tumoren ein Tumorregressionsgrad zugeordnet wurde. In diesem Zusammenhang sei jedoch erwähnt, dass das Vorhandensein von großflächigen Schaumzellaggregaten, ein eher zentrales Fibrosemuster und zytotoxische Gefäßveränderungen wie eine my- xohyaline Intimaproliferation oft mit atypischen reaktiv veränderten Endothelzellen, Teleangiektasien, organisierenden Thromben und obliterierender Endarteritis, jedoch signifikant häufiger bei neoadjuvant behandelten Tumoren im Vergleich zu primär resezierten Tumoren beobachtet wird [2].

Auf zellulärer Ebene können die residuellen Tumorzellen ein im Vergleich zu unbehandelten Tumoren (oder auch der korrespondierenden prätherapeutischen $\mathrm{Bi}$ opsie) oftmals weites, eosinophiles Zytoplasma mit Vakuolisierung oder onkozytärer Differenzierung aufweisen. Nukleäre Atypien wie Hyperchromasie, Pyknose, Karyorrhexis oder die Bildung großer, bizarrer Kerne sind ebenfalls häufige Befunde. Mitosen werden eher selten beobachtet. Diese Veränderungen können innerhalb von Tumoren auch heterogen ausgeprägt sein, sodass histologisch unauffällige Bereiche von Tumorinfiltrationen direkt neben derartig atypischen Drüsen oder Zellen mit signifikanten zytologischen Veränderungen beobachtet werden können [2]. Zudem zeigen sowohl das tumorale als auch das nichttumorale Stroma charakteristische Veränderungen, oftmals mit sogenannten bizarren Stromafibroblasten. Auch in nichtneoplastischem Nachbargewebe können therapieassoziierte Veränderungen wie Ödem und Entzündung be- 


\begin{tabular}{|c|c|c|c|}
\hline $\begin{array}{l}\text { Deskriptive } \\
\text { Regression }\end{array}$ & Mandard & AJCC & Becker \\
\hline \multirow[t]{2}{*}{ Komplett } & TRG 1 & TRG 0 & TRG 1a \\
\hline & $\begin{array}{l}\text { Keine residuellen Tu- } \\
\text { morzellen }\end{array}$ & Keine residuellen Tumorzellen & $\begin{array}{l}\text { Komplette Re- } \\
\text { gression }\end{array}$ \\
\hline \multirow[t]{2}{*}{ Subtotal } & TRG 2 & TRG 1 & TRG $1 b$ \\
\hline & Wenige Tumorzellen & $\begin{array}{l}\text { Einzelzellen oder kleine Tumor- } \\
\text { zellgruppen }\end{array}$ & $\begin{array}{l}<10 \% \text { Residual- } \\
\text { tumor }\end{array}$ \\
\hline \multirow[t]{2}{*}{ Partiell } & TRG 3 & TRG 2 & TRG 2 \\
\hline & $\begin{array}{l}\text { Mehr Fibrose als Resi- } \\
\text { dualtumor }\end{array}$ & $\begin{array}{l}\text { Residualtumor mit desmoplasti- } \\
\text { scher Reaktion }\end{array}$ & $\begin{array}{l}10-50 \% \text { Resi- } \\
\text { dualtumor }\end{array}$ \\
\hline \multirow{4}{*}{$\begin{array}{l}\text { Keine } \mathrm{Re}- \\
\text { gression }\end{array}$} & TRG 4 & \multirow{4}{*}{$\begin{array}{l}\text { TRG } 3 \\
\text { Minimale Regression }\end{array}$} & \multirow{4}{*}{$\begin{array}{l}\text { TRG } 3 \\
>50 \% \text { Residual } \\
\text { tumor }\end{array}$} \\
\hline & $\begin{array}{l}\text { Mehr Residualtumor als } \\
\text { Fibrose }\end{array}$ & & \\
\hline & TRG 5 & & \\
\hline & Keine Regression & & \\
\hline
\end{tabular}

\begin{tabular}{|c|c|c|}
\hline $\begin{array}{l}\text { Deskriptive } \\
\text { Regression }\end{array}$ & Dworak & Köln \\
\hline \multirow{2}{*}{$\begin{array}{l}\text { Keine Re- } \\
\text { gression }\end{array}$} & TRG 0 & TRG 1 \\
\hline & Keine Regression & $>50 \%$ vitale residuelle Tumorzellen \\
\hline \multirow[t]{4}{*}{ Partiell } & TRG 1 & \multirow[t]{2}{*}{-} \\
\hline & $\begin{array}{l}\text { Prädominanz von Tumorzellen mit signi- } \\
\text { fikanter Fibrose und/oder Vaskulopathie }\end{array}$ & \\
\hline & TRG 2 & TRG 2 \\
\hline & $\begin{array}{l}\text { Prädominanz der Fibrose mit vereinzelten } \\
\text { Tumorzellen (leicht histologisch zu sehen) }\end{array}$ & $\begin{array}{l}10-50 \% \text { vitale residuelle Tumorzel- } \\
\text { len }\end{array}$ \\
\hline \multirow[t]{2}{*}{ Subtotal } & TRG 3 & TRG 3 \\
\hline & $\begin{array}{l}\text { Nur ganz vereinzelt Tumorzellen in der } \\
\text { Fibrose mit oder ohne azellulärem Muzin }\end{array}$ & $<10 \%$ vitale residuelle Tumorzellen \\
\hline \multirow[t]{2}{*}{ Komplett } & TRG 4 & TRG 4 \\
\hline & Keine vitalen Tumorzellen & Komplette Regression \\
\hline
\end{tabular}

obachtet werden. Veränderungen in nichtneoplastischen Epithelien können denen von Tumorzellen ähneln (z.B. nukleärer Pleomorphismus, kondensiertes Chromatin und Eosinophilie). Solche Veränderungen von nichtneoplastischem Gewebe erscheinen gelegentlich besorgniserregend und können Schwierigkeiten in der Abgrenzung zu Karzinomen verursachen. Ein diagnostischer Fallstrick sind beispielsweise Atrophie und metaplastische Veränderungen nichtneoplastischer Drüsenstrukturen des Ösophagus und des Magens [2].

\section{Klassifikation der Tumorregression}

TRG-Systeme zielen darauf ab, das Ausmaß der regressiven Veränderungen nach zytotoxischer Behandlung zu kategorisieren, um potenzielle prognostische Informationen basierend auf objektiv bestimmbaren histopathologischen Befunden aufzuzeigen. Auf deskriptiver Ebene können Tumoren eine vollständige Regression oder verschiedene Mengen an Resttumor aufweisen: einige verstreute Resttumorzellen oder Gruppen innerhalb einer narbiger Fibrose (,subtotale Regression oder fast komplette Regression“), mehr als nur einzelne residuelle Tumorzellen oder Tumorzellgruppen („partielle Regression“) oder eine signifikante Tumormenge mit oder ohne Anzeichen regressiver Veränderungen ("keine signifikante Regression“; - Abb. 1). Wie im Abschnitt "Histopathologische Befunde nach neoadjuvanter Therapie" beschrieben, sind viele histopathologische Veränderungen nicht ganz spezifisch für eine Tumorregression. Daher beziehen sich die TRG-Systeme hauptsächlich auf einzelne, besser reproduzierbare Parameter.

Zur Klassifizierung des Tumorregressionsgrades lassen sich 2 Konzepte unterscheiden: einerseits die quantitative Abschätzung des Residualtumors (in Prozent oder deskriptiv), andererseits die Abschätzung des Verhältnisses zwischen Resttumor und regressiver Fibrose, die typischerweise auf einer Beschreibung basiert. Die TRG-Systeme nach Mandard [17] oder Dworak [6] sind Beispiele für TRG-Systeme, die sich auf das Verhältnis Tumor/Fibrose beziehen. Das BeckerSystem [2] oder das Kölner System [26] sowie das AJCC/CAP-System, das eine modifizierte Version des Ryan-Systems [25] darstellt, hingegen verwenden die Menge des Residualtumors bezogen auf das ursprüngliche Tumorareal. Eine Übersicht über verschiedenen TRG-Systeme und deren Kategorien geben - Tab. 1 und 2 .

\section{Prognostische Signifikanz der Tumorregression}

Zahlreiche Studien haben die prognostische Bedeutung des TRG untersucht. Die stärkste Evidenz für den Zusammenhang zwischen TRG und Patientenergebnis wurde bei Karzinomen des oberen Gastrointestinaltrakts beobachtet. Eine komplette Tumorregression ist im Allgemeinen mit einer sehr guten Prognose assoziiert $[1,13]$. Bei Magenkarzinomen wurde gezeigt, dass die prognostische Relevanz einer kompletten und subtotalen Regression (d. h. Mandard 1 und 2; Becker 1a und $1 \mathrm{~b}$ ) vergleichbar ist, sodass diese PatientInnen oft als "Responder" bezeichnet werden [1].Im Gegensatz dazu gibt es Daten für das Plattenepithel- und Adenokarzinom des Ösophagus, die nahelegen, dass bei diesen Tumoren eine subtotale und partielle Regression mit einer intermediären Prognose assoziiert ist [13]. Es ist daher nicht angebracht, 
die verschiedenen TRG-Systeme zu einem vereinfachten zweistufigen Klassifikationsschema mit "Respondern" und "Nichtrespondern" zu vereinfachen. Abhängig von der Fallzusammensetzung und den verwendeten statistischen Modellen waren TRG-Systeme neben dem Vorliegen von Lymphknotenmetastasen auch unabhängige prognostische Marker für das Überleben von Magen- und Ösophaguskarzinomen [1, 13], während in anderen Studien der Nachweis positiver Lymphknoten der einzige unabhängige Prognosefaktor war [5, 24]. Interessanterweise schnitt die ypT-Kategorie nach AJCC/UICC-Klassifikation häufig schlechter ab als die TRG- und yN-Kategorien [13, 24]. Im Hinblick auf das Regressionsmuster würde dies dafür sprechen, dass das Schrumpfen eines Tumors prognostisch nicht relevanter ist als eine Fragmentierung, wie für das Rektumkarzinom in einer Studie beschrieben [8]. Die gängigen Regressionsgraduierungssysteme berücksichtigen jedoch diese Unterschiede nicht.

Beim Rektumkarzinom sind die Daten zur klinischen Relevanz des TRG weniger aussagekräftig. Während eine vollständige Tumorregression konstant mit einem besseren krankheitsfreien und Gesamtüberleben assoziiert ist $[16,18]$, ist die Bedeutung einer subtotalen und partiellen Tumorregression weniger klar. Einige Kohortenstudien haben einen deutlichen prognostischen Einfluss einer partiellen Regression gezeigt [11, 16, 28], während andere Studien diese Ergebnisse nicht bestätigen [27]. Im Gegensatz zu Neoplasien des oberen Gastrointestinaltrakts erschwert beim Rektumkarzinom auch die hohe Variabilität verschiedener Behandlungsprotokolle die Vergleichbarkeit der Studien. Die wichtigsten prognostischen Faktoren für Rektumkarzinome mit oder ohne neoadjuvante Therapie sind der Status des zirkumferenziellen Resektionsrandes und - wie im oberen Gastrointestinaltrakt - das Vorhandensein oder Fehlen von Lymphknotenmetastasen. Da die Tumorregression jedoch stark mit diesen Faktoren assoziiert ist, ist erklärbar, warum der TRG in einigen Berechnungsmodellen die statistische Unabhängigkeit verliert [14].

\section{Tumorregression bei Lymph- knotenmetastasen}

Derzeit beziehen sich die TRG-Systeme auf regressive Veränderungen im primären Tumorareal („Tumorlager" oder „Tumorbett ${ }^{\prime \prime}$ ) und nicht auf Befunde in Lymphknoten oder Fernmetastasen. Eine Tumorregression kann jedoch auch in Lymphknoten, die metastasiert sind oder waren, beobachtet werden. Die histopathologischen Muster sind mit denen vergleichbar, die an primären Lokalisationen der Tumoren beobachtet wurden, wie z.B. Fibrose, resorptive Veränderungen, einschließlich histiozytärer Infiltrationen, und Fremdkörperreaktionen. Regressive und fibrotische Veränderungen, insbesondere in den mediastinalen Lymphknoten, können jedoch unabhängig von einer zytotoxischen Behandlung auftreten. Im Gegensatz dazu können kleine oder Mikrometastasen in Lymphknoten ohne signifikante Narbenbildung zurückgehen und der sichere Nachweis kleiner Metastasen vor der Behandlung ist mit bildgebenden Verfahren noch schwierig. Das Vorliegen von Lymphknotenmetastasen ist jedoch einer der wichtigsten prognostischen Faktoren bei gastrointestinalen Karzinomen nach neoadjuvanter Therapie und Operation [1, 7, 23]. Daher sollte bei Vorliegen einer Fibrose ohne Residualtumor in den ersten Schnittstufen eine weitere Aufarbeitung in Stufenschnitten in Betracht durchgeführt werden. Obwohl eine Graduierung der Tumorregression in Lymphknoten üblicherweise nicht durchgeführt wird, wurde vorgeschlagen, das Vorliegen oder Fehlen von Lymphknotenmetastasen unabhängig von regressiven Veränderungen in prognostische Stagingsysteme einzubeziehen [13]. Viele PathologInnen erwähnen in ihren Berichten ohnehin das Vorhandensein regressiver Veränderungen der Lymphknoten oder Lymphknotenmetastasen auch ohne weitere Regressionsgraduierung [31]. Bei Tumoren des oberen Gastrointestinaltraktes wurde gezeigt, dass eine komplette Regression in Lymphknotenmetastasen auch prognostisch einem Downstaging entspricht mit vergleichbaren Überlebensdaten wie bei ypN0-Tumoren ohne Regression, d.h. bereits prätherapeutisch negativem Lymphknotenstatus [21,
23]. Das Interobserveragreement für die Bewertung der Regression in Lymphknotenmetastasen wurde als substanziell beschrieben [23, 30]. Daten über die prognostische Bedeutung von regressiven Veränderungen an Lymphknoten mit noch nachweisbarem Residualtumor sind jedoch nicht konklusiv, sodass diesbezüglich weitere Studien nötig sind.

\section{Kritische Punkte der TRG}

Ein kritischer Punkt bei der Bestimmung eines TRG ist die Inter- und Intraobservervariabilität. Je nach Studiendesign wurde die Interobservervariabilität einer TRG als nicht zufriedenstellend [3] oder als substanziell beschrieben [10]. In Einzel- und Grenzfällen kann eine exakte Zuordnung zu einem TRG schwierig sein. In der täglichen Praxis sind die meisten Tumore jedoch klar einem bestimmten TRG zuordenbar [10, 12, 28]. Eine Quantifizierung des Residualtumors mittels digitaler Bildanalyse könnte hierbei hilfreich sein [20]. Derartige Techniken könnten auch dazu beitragen, andere morphologische Parameter wie die reine Bestimmung des Residualtumors als prognostisch relevant zu identifizieren, um diese dann in ein noch valideres Regressionsgraduierungssystem aufzunehmen. Hinsichtlich der Aufarbeitung hat sich gezeigt, dass die Bewertung des gesamten Tumorareals, wie sie in der Praxis durchgeführt wird, eine bessere Interobserverübereinstimmung aufweist, als nur die Bewertung eines repräsentativen Schnittes (wie es in einigen Interobserverstudien gemacht wurde) $[3,10]$.

Ein anderer kritischer Punkt ist die immer noch fehlende Standardisierung bei der histologischen Aufarbeitung und der abschließenden Verwendung der TRG-Systeme. Zwar wird in der Praxis das Tumorlagers zumindest bis zu einer gewissen Größe komplett eingebettet [31], allgemein gültige Standards existieren aber diesbezüglich nicht. In der Erstbeschreibung wurde zumindest für das Becker-, das Mandard- und das Ryan-System eine komplette Aufarbeitung des Tumorlagers beschrieben [2, 17, 22]. Hinsichtlich der Verwendung von TRG-Systemen existieren weltweit große regionale Unterschiede. Während in englischsprachigen Ländern, insbesondere den USA und Großbri- 
tannien, sehr homogen ein von der AJCC und der CAP auch empfohlenes modifiziertes Ryan-System für alle Tumorentitäten verwendet wird, wird in Europa neben dem AJCC/CAP System auch das MandardSystem, das Becker-System (insbesondere für Tumoren des oberen Gastrointestinaltraktes) und das Dworak-System (für Rektumkarzinome, insbesondere in deutschsprachigen Ländern) angewendet. In Japan werden die dortigen Richtlinien eingesetzt, die eine Quantifizierung mit anderen Schwellenwerten als z. B. beim BeckerSystem beinhalten [31]. Generell wird jedoch ein vierstufiges System von vielen Experten als ideal angesehen [19, 29, 31]. Im Gegensatz zur AJCC-TNM-Klassifikation, wo die Angabe eines TRG zumindest für das Rektumkarzinom empfohlen wird, ist in der UICC-TNM-Klassifikation die Tumorregression nicht als relevanter Parameter aufgeführt. Die kürzlich veröffentlichen Datensätze für standardisierte Tumorbefunde der International Collaboration of Cancer Reporting (ICCR) empfehlen ebenfalls nur für das Rektumkarzinom eine bestimmten TRG (die AJCC/CAP-TRG), während für das Ösophaguskarzinom die 3 am häufigsten verwendeten TRGs (Mandard, Becker, AJCC/CAP) als Möglichkeiten vorgestellt werden. Beim Magenkarzinom wird in einem Kommentar auf diese Varianten eingegangen, im Haupttext jedoch ebenfalls das AJCC/CAP-System empfohlen [9].

\section{Schlussfolgerung und Ausblick}

Die Tumorregressionsgraduierung ist als morphologischer und prognostischer Parameter in der pathologischen Aufarbeitung und Befundung von Resektaten von Tumoren des Gastrointestinaltraktes nach neoadjuvanter Therapie fest etabliert. Historisch und geografisch bedingt existieren eine Reihe von fast gleichwertigen Systemen, wobei eine verbindliche Empfehlung darüber, welches System verwendet werden sollte (und auch für welche Tumore), noch aussteht. Es ist deshalb sinnvoll, bei der Angabe eines Regressionsgrades den Namen des verwendeten Systems und im Idealfall auch die wörtliche Bedeutung anzugeben, um Fehlinterpretationen in der klinischen und wissenschaftlichen Umsetzung zu vermei- den. Eine internationale Standardisierung der Bewertung der TRG ist jedoch nach wie vor wünschenswert.

In den letzten Jahren wurden neben der Weiterentwicklung der konventionellen Radiochemotherapie- und Chemotherapieschemata für gastrointestinale Tumoren auch neue Therapieansätze für Tumorbehandlungen vorgestellt, wie zum Beispiel die zielgerichtete Anti-HER2-Therapie bei Adenokarzinomen des oberen Gastrointestinaltrakts oder zuletzt die Einführung der Immuncheckpoint-Inhibition [15]. Über die Gewebeveränderungen, die sich aus diesen neuen Behandlungsstrategien ergeben, ist jedoch wenig bekannt. Eine sorgfältige histopathologische Untersuchung des posttherapeutischen Gewebes kann - wie bei den initialen Beschreibungen der verschiedenen Regressionsgraduierungen - somit wichtige Aspekte der Wirkungen und Resistenzmechanismen dieser neuen Medikamente liefern. Inwieweit sich dies auf eine Regressionsgraduierung für künftig zu erwartenden Therapien auswirken wird, muss sorgfältig erarbeitet werden.

\section{Fazit für die Praxis}

- Die makroskopische und histopathologische Aufarbeitung von Resektaten gastrointestinaler Karzinome nach neoadjuvanter Therapie sollte standardisiert durchgeführt werden mit einer im Idealfall kompletten Untersuchung des ursprünglichen Tumorareals.

- Die Angabe eines Tumorregressionsgrades im histopathologischen Befund wird empfohlen.

- Es existieren verschiedene, prognostisch fast gleichwertige Systeme für die Tumorregressionsgraduierung, wobei es aktuell noch keine fest verbindliche Empfehlung für die Verwendung eines speziellen Systems gibt.

- Die Rolle der Regression in Lymphknoten muss noch weiter untersucht werden.

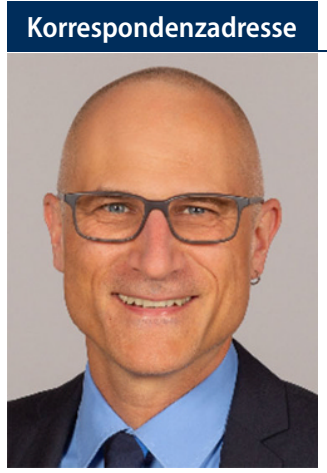

Univ.-Prof. Dr. Rupert Langer

Klinisches Institut für Pathologie und Molekularpathologie, Kepler Universitätsklinikum und Johannes Kepler Universität

Krankenhausstraße 9, 4021 Linz, Österreich

Rupert.Langer@kepleruniklinikum.at

Funding. Open access funding provided by Johannes Kepler University Linz.

\section{Einhaltung ethischer Richtlinien}

Interessenkonflikt. D. Liu und R. Langer geben an, dass kein Interessenkonflikt besteht.

Für diesen Beitrag wurden von den Autoren keine Studien an Menschen oder Tieren durchgeführt. Für die aufgeführten Studien gelten die jeweils dort angegebenen ethischen Richtlinien.

Open Access. Dieser Artikel wird unter der Creative Commons Namensnennung 4.0 International Lizenz veröffentlicht, welche die Nutzung, Vervielfältigung, Bearbeitung, Verbreitung und Wiedergabe in jeglichem Medium und Format erlaubt, sofern Sie den/die ursprünglichen Autor(en) und die Quelle ordnungsgemäß nennen, einen Link zur Creative Commons Lizenz beifügen und angeben, ob Änderungen vorgenommen wurden.

Die in diesem Artikel enthaltenen Bilder und sonstiges Drittmaterial unterliegen ebenfalls der genannten Creative Commons Lizenz, sofern sich aus der Abbildungslegende nichts anderes ergibt. Sofern das betreffende Material nicht unter der genannten Creative Commons Lizenz steht und die betreffende Handlung nicht nach gesetzlichen Vorschriften erlaubt ist, ist für die oben aufgeführten Weiterverwendungen des Materials die Einwilligung des jeweiligen Rechteinhabers einzuholen.

Weitere Details zur Lizenz entnehmen Sie bitte der Lizenzinformation auf http://creativecommons.org/ licenses/by/4.0/deed.de.

\section{Literatur}

1. Becker K, Langer R, Reim D et al (2011) Significance of histopathological tumor regression after neoadjuvant chemotherapy in gastric adenocarcinomas: a summary of 480 cases. Ann Surg 253:934-939

2. Becker K, Mueller JD, Schulmacher C et al (2003) Histomorphology and grading of regression 
in gastric carcinoma treated with neoadjuvant chemotherapy. Cancer 98:1521-1530

3. Chetty R, Gill P, Govender D et al (2012) International study group on rectal cancer regression grading: interobserver variability with commonly used regression grading systems. Hum Pathol 43:1917-1923

4. Cunningham D, Allum WH, Stenning SP et al (2006) Perioperative chemotherapy versus surgery alone for resectable gastroesophageal cancer. N Engl J Med 355:11-20

5. Cunningham D, Stenning SP, Smyth EC et al (2017) Peri-operative chemotherapy with or without bevacizumab in operable oesophagogastric adenocarcinoma (UK medical research council ST03): primary analysis results of a multicentre, open-label, randomised phase 2-3 trial. Lancet Oncol 18:357-370

6. Dworak O, Keilholz L, Hoffmann A (1997) Pathological features of rectal cancer after preoperative radiochemotherapy. Int J Colorectal Dis 12:19-23

7. Fokas E, Liersch T, Fietkau R et al (2014) Tumor regression grading after preoperative chemoradiotherapy for locally advanced rectal carcinoma revisited: updated results of the CAO/ARO/AIO-94 trial. JClin Oncol 32:1554-1562

8. Hav M, Libbrecht L, Geboes Ketal (2015) Prognostic value of tumor shrinkage versus fragmentation following radiochemotherapy and surgery for rectal cancer. Virchows Arch 466:517-523

9. International Collaboration On Cancer Reporting (2021) Digestive tract. http://www. Iccr-Cancer.Org/Datasets/Published-Datasets/ Digestive-Tract.Zugegriffen: 14.09.2021

10. Karamitopoulou E, Thies S, Zlobec I et al (2014) Assessment of tumor regression of esophageal adenocarcinomas after neoadjuvant chemotherapy: comparison of 2 commonly used scoring approaches. Am J Surg Pathol 38:1551-1556

11. Kim JY, Park IJ, Hong SM et al (2015) Is pathologic near-total regression an appropriate indicator of a good response to preoperative chemoradiotherapy based on oncologic outcome of disease? Medicine 94:e2257

12. Kim SH, Chang HJ, Kim DY et al (2016) What is the ideal tumor regression grading system in rectal cancer patients after preoperative chemoradiotherapy? Cancer Res Treat 48:998-1009

13. Langer R, Becker K, Zlobec I et al (2014) A multifactorial histopathologic score for the prediction of prognosis of resected esophageal adenocarcinomas afterneoadjuvant chemotherapy. Ann Surg Oncol 21:915-921

14. Lee YC, Hsieh CC, Chuang JP (2013) Prognostic significance of partial tumor regression after preoperative chemoradiotherapy for rectal cancer: a meta-analysis. Dis Colon Rectum 56:1093-1101

15. Lordick F, Shitara K, Janjigian YY (2017) New agents on the horizon in gastric cancer. Ann Oncol 28:1767-1775

16. Mace AG, Pai RK, Stocchi L et al (2015) American joint committee on cancer and college of American pathologists regression grade: a new prognostic factor in rectal cancer. Dis Colon Rectum 58:32-44

17. Mandard AM, Dalibard F, Mandard JC et al (1994) Pathologic assessment of tumor regression after preoperative chemoradiotherapy of esophageal carcinoma. Clinicopathologic correlations. Cancer 73:2680-2686

18. Martin ST, Heneghan HM, Winter DC (2012) Systematic review and meta-analysis of outcomes following pathological complete response to

\section{Grading of tumor regression of gastrointestinal carcinomas after neoadjuvant therapy}

Pre- or perioperative chemo- or radiochemotherapy and subsequent resection is the standard therapy for locally advanced esophageal, gastric, and rectal cancer. A tumor regression grading (TRG; also tumor regression grade) categorizes the extent of the regressive changes after a neoadjuvant treatment. There are several TRG systems for gastrointestinal carcinomas that relate either to the extent of the therapy-induced fibrosis in relation to the residual tumor or the estimated proportion of the residual tumor in the area of the former tumor area. An ideal TRG system shows significant interobserver agreement and offers relevant prognostic information - in most cases a complete or almost complete regression after neoadjuvant therapy is associated with an improved prognosis. In this review, the most commonly used TRG systems for gastrointestinal carcinomas are presented and discussed. In addition, current issues such as the standardization of TRG and the subject of regression in lymph node metastases in the context of a TRG system are discussed.

\section{Keywords}

Gastrointestinal neoplasms · Histopathology · Tumor regression grading · Observer variation . Reference standards

neoadjuvant chemoradiotherapy for rectal cancer. BrJSurg 99:918-928

19. Minsky BD, Rodel C (2014) Identifying the most predictive post-chemoradiation TRG system for rectal cancer. J Natl Cancer Inst 106(10):dju285. https://doi.org/10.1093/jnci/dju285

20. Nakamura K, Kuwata T, Shimoda T et al (2015) Determination of the optimal cutoff percentage of residual tumors to define the pathological response rate for gastric cancer treated with preoperative therapy (JCOG1004-A). Gastric Cancer 18:597-604

21. Philippron A, Bollschweiler E, Kunikata $A$ et al (2016) Prognostic relevance of lymph node regression after neoadjuvant chemoradiation for esophageal cancer. Semin Thorac Cardiovasc Surg 28:549-558

22. Quirke P, Durdey P, Dixon MF et al (1986) Local recurrence of rectal adenocarcinoma due to inadequate surgical resection. Histopathological study of lateral tumour spread and surgical excision. Lancet 2:996-999

23. Reim D, Novotny A, Friess Hetal (2020) Significance of tumour regression in lymph node metastases of gastric and gastro-oesophageal junction adenocarcinomas. JPathol Clin Res 6:263-272

24. Rizk NP, Venkatraman E, Bains MS et al (2007) American joint committee on cancer staging system does not accurately predict survival in patients receiving multimodality therapy for esophageal adenocarcinoma. J Clin Oncol 25:507-512

25. Ryan R, Gibbons D, Hyland JM et al (2005) Pathological response following long-course neoadjuvant chemoradiotherapy for locally advanced rectal cancer. Histopathology 47:141-146

26. Schneider PM, Baldus SE, Metzger R et al (2005) Histomorphologic tumor regression and lymph node metastases determine prognosis following neoadjuvant radiochemotherapy for esophageal cancer: implications for response classification. Ann Surg 242:684-692

27. Swellengrebel HA, Bosch SL, Cats A et al (2014) Tumour regression grading after chemoradiotherapy for locally advanced rectal cancer: a near pathologic complete response does not translate into good clinical outcome. Radiother Oncol 112:44-51

28. Trakarnsanga A, Gonen M, Shia J et al (2014) Comparison of tumor regression grade systems for locally advanced rectal cancer after multimodality treatment. J Natl Cancer Inst 106(10):dju248. https://doi.org/10.1093/jnci/dju248

29. Tsekrekos A, Detlefsen S, Riddell R et al (2019) Histopathologic tumor regression grading in patients with gastric carcinoma submitted to neoadjuvant treatment: results of a Delphi survey. Hum Pathol 84:26-34

30. Tsekrekos A, Vieth $M$, Ndegwa $N$ et al (2021) Interobserver agreement of a gastric adenocarcinoma tumor regression grading system that incorporates assessment of lymph nodes. Hum Pathol 116:94-101. https://doi.org/10.1016/j. humpath.2021.07.003

31. Westerhoff M, Osecky M, Langer R (2020) Varying practices in tumor regression grading of gastrointestinal carcinomas after neoadjuvant therapy: results of an international survey. Mod Pathol 33:676-689 